\title{
L'épisode neigeux de décembre 1990 dans la région Rhône-Alpes
}

\author{
J.C. Deblaere, Météo-France - Lyon
}

\section{Introduction}

La neige, $\ldots$ on l'avait presque oubliée tant elle était devenue rare depuis deux à trois ans.

Pourtant ce dimanche 9 au matin, elle est bien là, couvrant tout sur une bonne partie du Centre-Est de la France.

Le phénomène prend même des allures de catastrophe dans les départements de l'Ain, de l'Isère, du Rhône. La situation est moins critique dans la Loire, le Nord de la Drôme et de l'Ardèche, l'Ouest de la Savoie.

Ce sera notre domaine de travail, domaine qui regroupe les régions ayant connu un enneigement tout à fait inhabituel. Il faut noter que les chutes de neige ont été très abondantes sur certains reliefs, par exemple le Bugey. Mais là le phénomène n'est pas inattendu, chacun est mieux préparé à cette éventualité et les conséquences s'en trouvent minimisées.

\section{Le réseau de mesures}

Les données proviennent :

- des stations de la Météorologie Nationale (8 sur le domaine retenu);

- des postes climatologiques, tenus par du personnel quasi bénévole, effectuant le plus souvent un seul relevé par jour.

La trop faible densité du réseau de postes utilisables pour la mesure de la neige (une cinquantaine de postes malgré tout) ne permet pas d'exclure quelques imprécisions dans le tracé des cartes ou histogrammes. Ces documents sont destinés pour l'essentiel à rendre compte de l'ampleur du phénomène et constituent nullement une base d'information précise. Et puis la neige est une forme de précipitations. On peut donc s'attendre à des variations spatiotemporelles analogues à celles que l'on rencontre pour la

\section{Snowfalls in Rhône-Alps region (France) december 1990}

Nearly all the activities of the RHONE-ALPS Region were disturbed by unusual snowfalls.

This was an unusual phenomen because of its location (essentially low-altitude regions) as well as by its importance.

Classically it was caused by the arrival of hot and humid air over a film of cold air and by disturbances being stuck before the Alps. It was a matter of a few hours' time and of a few degrees.

Following the study of the meteorological situation and the distribution of the snow in time and space, it was necessary to handle the problems of excess load to together with the frequency of such a phenomen since it was a memorable event. 
pluie. Avec quelques complications supplémentaires dues au vent, aux petites variations de température qui peuvent se traduire par un changement d'état, ...

\section{La situation météorologique}

L'étude de la situation météorologique est réalisée à l'aide des cartes " analyse en surface» et "surface $500 \mathrm{hPa} *$ ».

\subsection{Pour comprendre ces documents}

Analyse en surface

Chaque carte reproduit la situation " au sol " à 13 heures (heure locale).

Apparaissent sur ces cartes :

- les conditions météorologiques pour une sélection de postes. Elles sont représentées sous forme symbolique ;
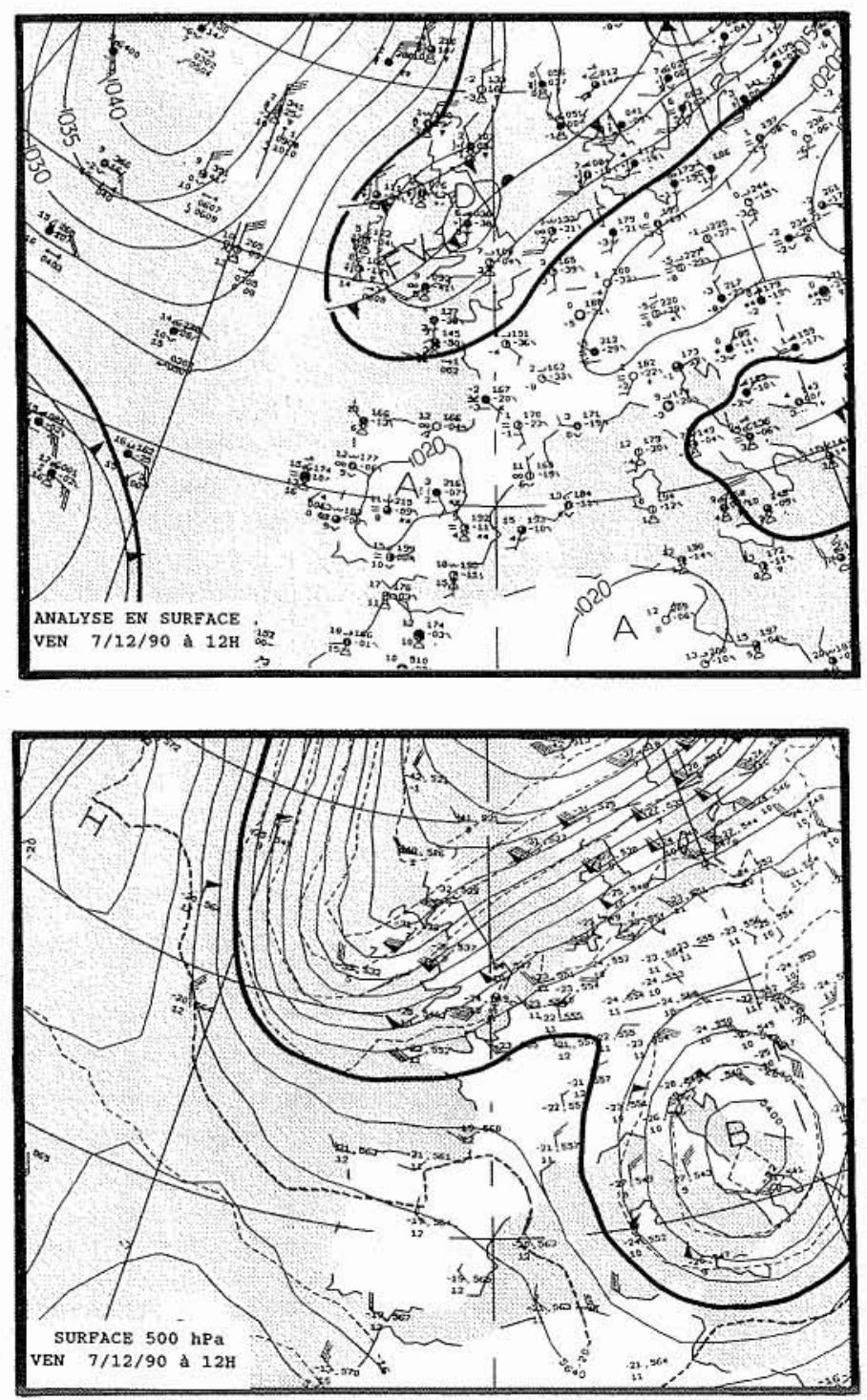

- les isobares cotées de 5 en $5 \mathrm{hPa}$. Il s'agit des lignes joignant les points où la pression atmosphérique, réduite au niveau de la mer est la même à un instant donné. Les isobares délimitent des zones de basse pression (dépression notée D) ou de haute pression (anticyclone noté A);

- Les limites de masses d'air matérialisées par des fronts.

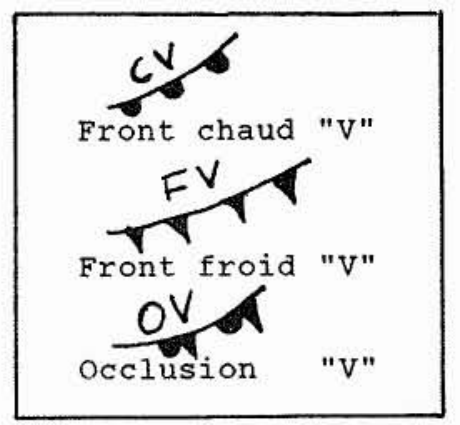

\section{Analyse en altitude}

Pour des raisons d'ordre pratique, un niveau de l'atmosphère est repéré par sa pression, plutôt que par son altitude. La référence est donc une surface isobare dont on étudie la configuration à l'aide :

- des lignes de niveau, ce sont les isohypses;

- des lignes d'égale température, ce sont les isothermes, représentées ici en pointillés.

\subsection{La situation... le vendredi 7 décembre}

\section{En surface}

Voilà déjà plusieurs jours que la France " est protégée " par un puissant anticyclone centré sur le Nord de la France.

Depuis mardi les prévisions font état de chutes de neige pour samedi. Difficile à admettre tant il fait beau! Mais rapidement tout se dégrade : l'air froid jusqu'alors immobile au large de la Norvège progresse vers le Pays de Galles. Le front froid «V » qui lui est associé approche des côtes de la Manche, apportant douceur et nuages.

\section{En altitude}

La surface $500 \mathrm{HPa}$ révèle deux éléments importants :

- des basses pressions sur l'Italie. Elles migrent vers l'Est en se comblant : elles ne nous intéressent plus;

- un talweg très accusé, axé du Sud de l'Irlande à la Finlande. La circulation rapide à l'avant de ce talweg ne peut pas résister longtemps au courant de Nord qui entraîne l'air froid coincé entre l'Islande et la Norvège.

\footnotetext{
${ }^{*} \mathrm{hPa}=$ hecto Pascal.
} 


\subsection{La situation... le samedi 8 décembre}

Tout va vite, très vite même. Il n'y a plus aucun lien entre la situation de ce jour et celle de la veille.

Ainsi en surface ; l'anticyclone a été balayé, remplacé par une dépression assez profonde.

Le front froid " $\mathrm{V}$ » est entraîné rapidement vers le Sud.

A 12 heures, il se situe du Portugal à la Hollande, tout en marquant quelques ondulations.

A 21 heures et malgré de nombreuses contorsions on le retrouve du Roussillon au Rouergue et au Jura. Du rarement vu.

Tandis qu'en altitude, la « goutte froide » emprisonne de l'air dont la température avoisine - 40 degrés et ce, presque au-dessus de la Bretagne.

A 0 heure la radio sondage de Lyon-Satolas porte encore l'empreinte du séjour de l'anticyclone: une remarquable inversion de température : -3 degrés près du sol, presque 3 degrés 150 mètres plus haut. Et bien, 12 heures plus tard l'inversion a disparu.

\subsection{La situation... les jours suivants}

Dimanche 9, lundi 10 et mardi 11, l'air froid d'altitude se déplace peu, passant de la Bretagne à la Provence. Coupé de son alimentation froide, du moins à nos latitudes il génère des phénomènes de moins en moins virulents.

Ainsi la perturbation " $\mathrm{V}$ " se décale vers l'Est tout en perdant de son activité. Elle nous intéresse seulement par une occlusion qui s'étire de l'Europe du Nord au CentreEst de la France.

Mercredi 12, une nouvelle perturbation « $\mathrm{A}$ » traverse la France du Nord au Sud. En altitude, l'air froid se déplace maintenant plus à l'Est, du Danemark vers la Sicile.

Jeudi 13 et vendredi 14 les conditions deviennent anticycloniques sur l'Angleterre. Il fait froid mais tout rentre lentement dans l'ordre.

\subsection{Les conséquences}

Mais qu'est-ce qui s'est passé ce samedi 8 décembre?

Dans la nuit du 7 au 8 il fait encore froid. Samedi matin le vent tourne au Sud, se renforce, atteignant presque $60 \mathrm{~km} / \mathrm{h}$ en pointe. Les températures s'élèvent, on gagne au moins 5 degrés par rapport à la veille.

Il fait encore 3 à 4 degrés quand débutent les premières pluies importantes (après 18 heures à Lyon).

Peu après le vent s'oriente au Nord, la baisse sensible de température ne se produit qu'en milieu de nuit: la neige succède à la pluie.

A l'Ouest et au Sud de Lyon, parce qu'il fait un peu plus chaud, la neige arrivera plus tard. Le phénomène s'inverse quand on se déplace vers l'Est.

\section{Et les jours suivants?}

Il va neiger de façon plus ou moins continue jusqu'au mardi 11 au matin et cela presque partout.
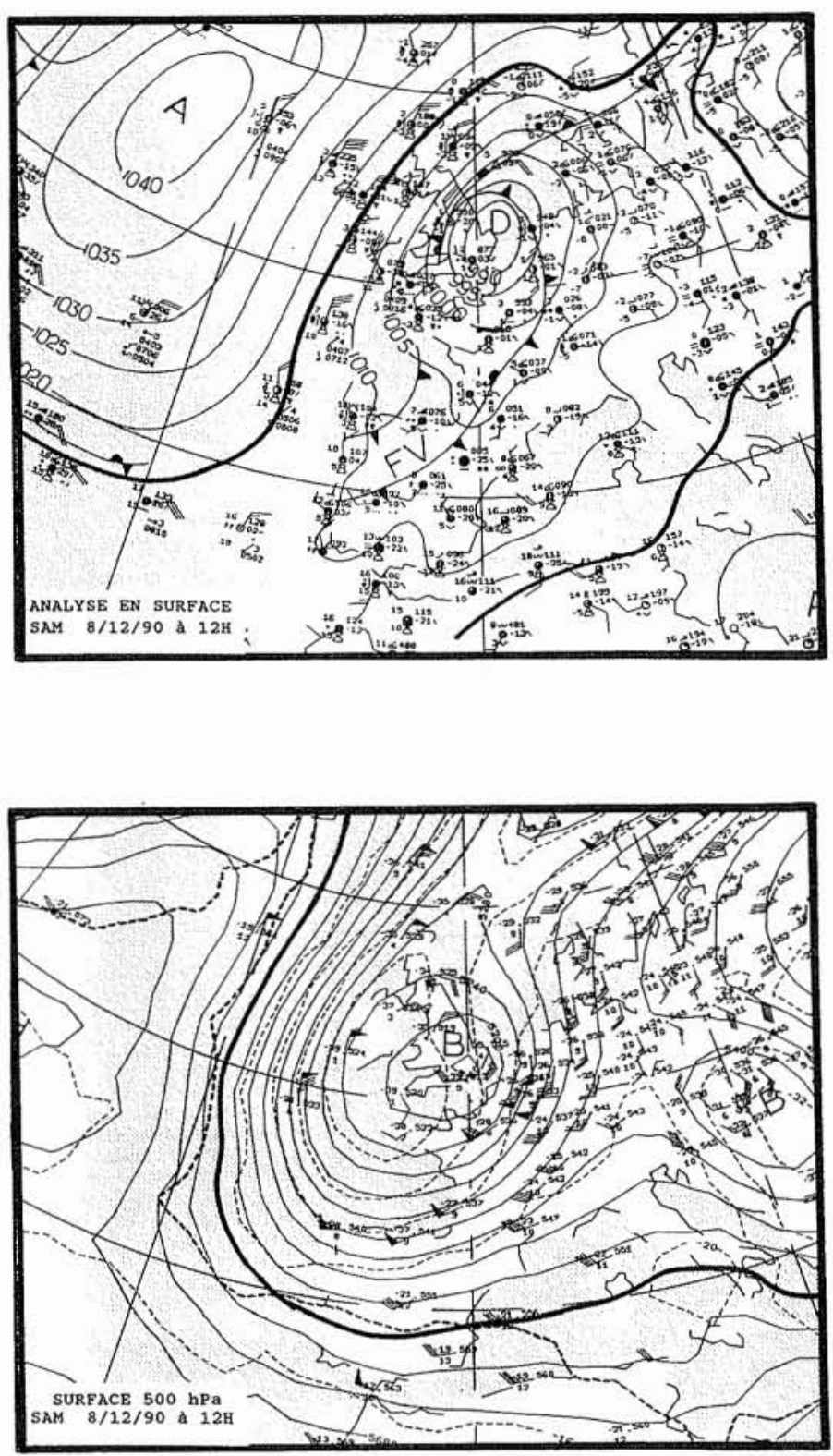

Les températures restent voisines de zéro degré et le vent est faible, heureusement.

Pour la journée du 11 c'est différent : peu ou pas de neige, les températures s'abaissent et le vent se renforce. Les chutes de neige reprennent de façon plus discontinue et éparse le 12 et le 13. Le vent se maintient au secteur Nord et reste assez fort.

Pratiquement pas de neige le 14 et cet épisode se termine par quelques flocons ici et là dans la journée du samedi 15 . 


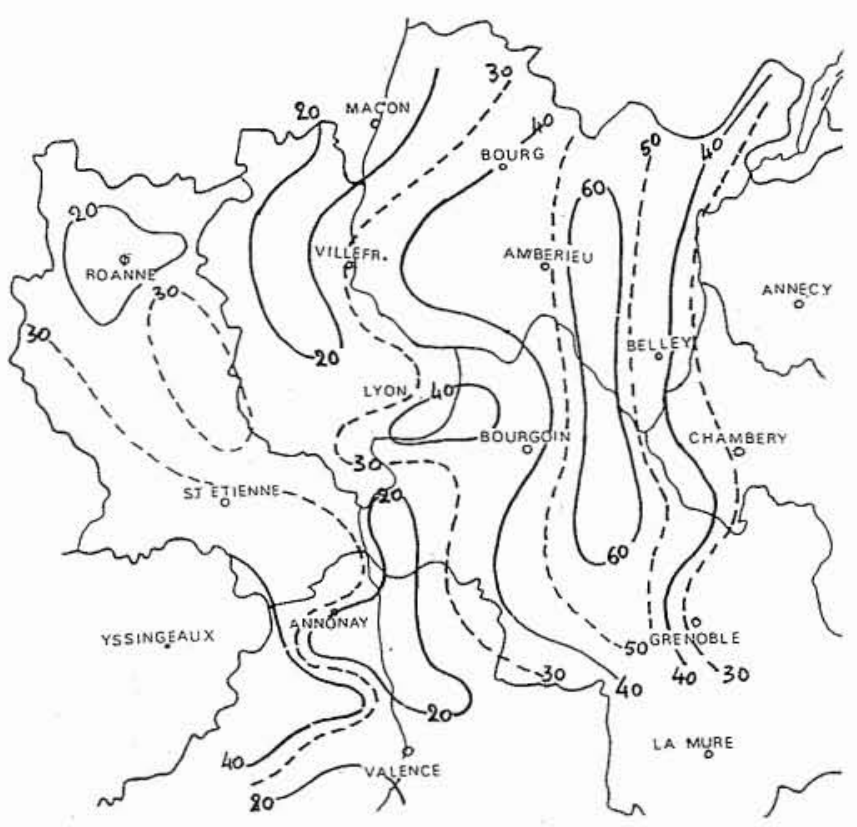

le 10 décembre 1990 au matin

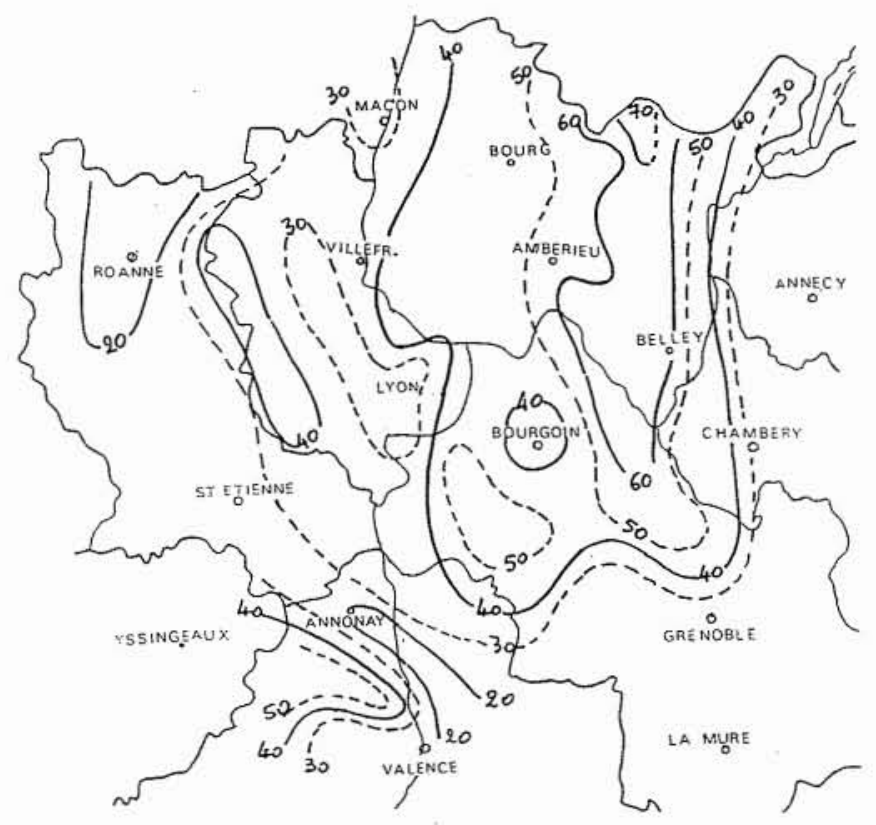

le 12 décembre 1990 au matin

Epaisseur de la couche de neige $(\mathrm{cm})$.

\section{Hauteurs de neige}

\subsection{Répartition dans l'espace}

L'épaisseur du manteau neigeux est présentée sur fond de carte couvrant la majeure partie de la région Rhône-Alpes.

\section{Le lundi 10 au matin}

Il neige depuis plus de 30 heures et l'épaisseur est déjà appréciable.

L'Ain et l'Isère, ainsi que l'Est lyonnais sont parmi les régions les plus touchées. La couche de neige dépasse déjà $40 \mathrm{~cm}$ sur les $2 / 3$ de cette zone, atteignant même une soixantaine de $\mathrm{cm}$ d'Oyonnax aux environs de la Tour du Pin.

Quelques régions sont plus épargnées : autour de Roanne, les premières pentes du Beaujolais, le Rhône moyen.

En Ardèche, dans le secteur Lamastre-Colombier-LeJeune la couche de neige dépasse $40 \mathrm{~cm}$.

Autre particularité, à la Chartreuse de Benonces dans l'Ain, ce matin là il faut dégager un mètre de neige. Bien sûr on est à près de 1000 mètres mais on n'observe rien d'approchant sur les massifs voisins.

\section{Le mercredi 12 au matin}

La couche de neige atteint son épaisseur maximale sur la majeure partie du domaine.

On retrouve les grands traits de la situation du 10 au matin. De façon schématique l'épaisseur du manteau neigeux a augmenté de 7 à $12 \mathrm{~cm}$ depuis le 10 .

Quelques exceptions toutefois:

- peu de changements autour de Roanne et de Valence à Annonay,

- forte augmentation à Colombier-Le-Jeune où la couche atteint $60 \mathrm{~cm}$.

A Benonces on mesure près de $1,30 \mathrm{~m}$ et ce n'est pas fini puisque le 14 au matin on atteindra $1,60 \mathrm{~m}$.

\subsection{Variations dans le temps}

Elles sont illustrées par une série d'histogrammes présentant le résultat de mesures effectuées le matin du jour indiqué.

- L'épaisseur de neige au sol.

- La hauteur de neige fraîche cumulée depuis le 8 au matin.

Sans détailler à l'excès on met en évidence 3 types d'évolution du manteau neigeux au cours de cette période. 

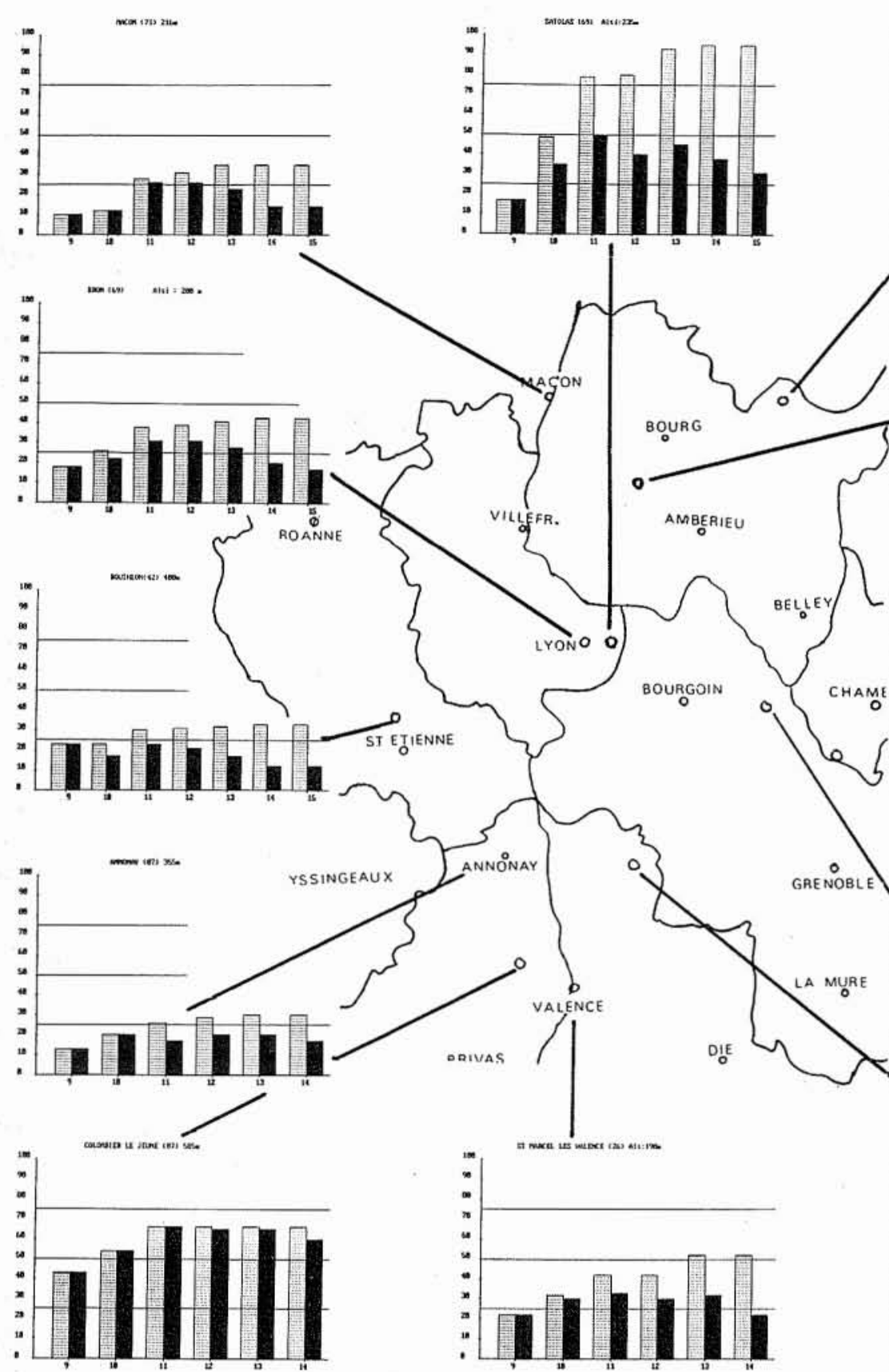

Hauteur cumulée de neige fraîche

Hauteur de neige au sol

\section{Type BRON (Rhône):}

Valence, Mâcon, St-Etienne, appartiennent à ce groupe.

Le cumul de neige fraîche augmente jusqu'au $14 / 15$ et l'épaisseur du manteau neigeux passe par un maximum bien marqué, le 12 .

La neige subit un fort tassement, à tel point qu'en fin de période l'épaisseur du manteau neigeux ne représente plus que le tiers de la hauteur de neige cumulée.

\section{Type OYONNAX (Ain) :}

On trouve dans ce groupe la plupart des postes situés dans un fuseau délimité par Satolas et Grenoble.
La hauteur de neige cumulée dépasse souvent $80 \mathrm{~cm}$, l'épaisseur du manteau neigeux est supérieure à $50 \mathrm{~cm}$.

Les chutes de neige se poursuivent jusqu'en fin de période. Si bien que le tassement du manteau neigeux est largement compensé par l'apport de neige fraîche, et son épaisseur décroît peu.

\section{Type COLOMBIER (Ardèche):}

Il neige peu ou pas après le 11 . C'est le moment où l'on observe l'épaisseur maximale du manteau neigeux.

Cette épaisseur varie très peu les jours suivants et le tassement est plutôt faible.

L'évolution est très voisine à Annonay. 


\section{Propriétés physiques du manteau neigeux}

Nous avons observé que l'épaisseur de la couche de neige était le plus souvent bien inférieure à la hauteur de neige fraîche cumulée.

A cela deux raisons principales: la fonte et la métamorphose.

Les phénomènes sont en partie liés et se traduisent par une modification des propriétés physiques, sensible au niveau de l'aspect, de la masse volumique, ...

Au cours de cet épisode les chutes de neige ont, outre la gêne occasionnée à la circulation, causé de gros dommages aux superstructures: lignes électriques coupées sous l'effet de la neige "collante», effondrements de toitures.

Faute d'informations précises et suffisamment nombreuses il n'est pas possible de tracer des cartes de masse volumique ou de surcharge.

On peut tout de même avancer quelques chiffres.

Pour la masse volumique :

de 80 à $140 \mathrm{~kg} / \mathrm{m}^{3}$ au cours des deux premiers jours. Ensuite on dépassera $200 \mathrm{~kg} / \mathrm{m}^{3}$, principalement dans l'Ain, l'Isère et très localement dans l'Est lyonnais.

A partir de l'équivalent en eau (c'est la hauteur d'eau recueillie en faisant fondre la neige) on peut évaluer la charge maximale supportée par une surface horizontale grâce à la relation simple :

$$
1 \mathrm{~mm} \text { d'eau }=1 \text { litre } / \mathrm{m}^{2}=1 \mathrm{~kg} / \mathrm{m}^{2}
$$

Ce qui permet d'estimer que :

A Nantua, La Tour du Pin, la charge n'a pas dépassé $115 \mathrm{~kg} / \mathrm{m}^{2}$.

A Bron, $50 \mathrm{~kg} / \mathrm{m}^{2}$ constitue bien un maximum.

Ces valeurs sont à minorer du poids de l'eau qui a fondu dans l'intervalle, et voici qu'apparaît l'inconnue majeure.

L'examen des températures montre que la fonte de neige au sol a été assez faible sauf peut-être dans les régions de plaine allant de la vallée du Rhône au Val de Saône et à la Loire.

Par contre la fonte sur les toitures est fortement dépendante du niveau d'isolation et là le phénomène n'est pas négligeable.

\section{Fréquence du phénomène}

Sur les départements de la Loire et le Nord du Rhône cet épisode n'est pas tout à fait exceptionnel.

Ainsi à Mâcon, depuis 1944 on avait déjà dépassé :

- 6 fois une épaisseur de $20 \mathrm{~cm}$

- 2 fois une épaisseur de $25 \mathrm{~cm}$.

Il est raisonnable d'estimer qu'un tel événement se produit en moyenne tous les 14 à 18 ans.

Dans la banlieue lyonnaise, en se basant sur les mesures effectuées à BRON depuis une cinquantaine d'années, on avait déjà dépassé :

- 5 fois une épaisseur de $20 \mathrm{~cm}$

-1 fois une épaisseur de $30 \mathrm{~cm}$.

Ce qui nous conduit à une fréquence moyenne pour cet épisode de une fois tous les 25 ans.

Dans l'Ain et l'Isère, les données anciennes sont plus clairsemées. Les relevés de St-Etienne-de-St-Geoirs (Isère) font apparaitre que depuis bientôt 50 ans on n'avait pas dépassé une épaisseur de $45 \mathrm{~cm}$ (en décembre 1970).

Il y a lieu de penser que des épaisseurs supérieures à $60 \mathrm{~cm}$ (c'était le cas dans cette région) ne se mesurent en moyenne qu'une fois tous les 40 à 60 ans.

\section{Remerciements}

- aux observateurs qui assurent les mesures avec dévouement et compétence,

- aux personnels de Météo-France qui dans chacun des départements concernés ont réalisé la collecte et le contrôle des données,

- à J.P. NAvarre (Centre d'Etudes de la neige) et Brigitte BENECH (Division de Prévision-Paris),

- aux personnels de la Division de Climatologie à Lyon qui ont contribué à la réalisation de cet article. 